\title{
On the influence of clustering processes in the liquid structure on Raman scattering
}

\author{
Gennady Melnikov ${ }^{1, *}$, Nikolay Ignatenko ${ }^{1}$, Lyudmila Petrova ${ }^{1}$, Olga Manzhos $^{1}$ and Andrey \\ Gromkov $^{1}$ \\ ${ }^{1}$ Southwest State University, 305040 Kursk, Russia
}

\begin{abstract}
A mathematical relation has been obtained that makes it possible to calculate the polarizability of a polyatomic molecule in the structure of a cluster. It is shown that the scattered frequencies in the Raman effect are proportional to the square root of the number of particles in the most probable (or average) cluster in the liquid structure. The appearance of frequencies in the far part of the Raman spectrum region is caused by the processes of intermolecular interactions in clusters and the processes of disintegration or formation of cluster systems in the structure of disordered condensed media. According to the proposed model and experimental data in the frequency range $20-1300 \mathrm{~cm}^{-1}$, it has been carried out the comparison of the values of the calculated frequencies of the Raman spectrum and their mutual position, which has shown the adequacy of the proposed model. The cluster model of liquid structure and the methods of mathematical statistics and statistical thermodynamics make it possible to expand the capabilities of the classical theory of Raman scattering in liquids and to predict the position of spectral bands in Raman spectra in the far long-wavelength region of the spectrum. It is revealed that the formation and breakdown of the most probable clusters is associated with the correlations of the most stable clusters (in terms of the number of particles) in a condensed medium with the Fibonacci numbers.
\end{abstract}

\section{Introduction}

At present the classical theory of Raman scattering (Raman effect) is well tested and generally agrees with experimental data, the essence of this theory is as follows. The dipole moment of a molecule in a substance is induced by an electric field of an incident electromagnetic wave, which distorts the general electron cloud of the molecule, and the induced dipole moment will be [1-5]

$$
\mu=\alpha E_{0} \cos \left(\omega_{0} t\right) .
$$

The molecular system has its own vibration modes $Q_{k}$, which are usually described by the relations

\footnotetext{
*Corresponding author: melnikovga@mail.ru
} 


$$
Q_{k}=Q_{k}^{0} \cos \left(\omega_{k} t\right)
$$

Such vibrations can affect the polarizability of the molecule, which is determined by the formula [1-5]

$$
\alpha=\alpha_{0}+\frac{\partial \alpha}{\partial Q_{k}^{0}} \cdot Q_{k}+\ldots
$$

For the induced dipole moment of the molecule, within the framework of the classical theory of the Raman effect, we obtain the relation

$$
\mu=\alpha_{0} E_{0} \cos (\omega t)+\frac{1}{2} E_{0} \cdot Q_{k}^{0} \frac{\partial \alpha}{\partial Q_{k}}\left(\left[\cos \left(\omega+\omega_{k}\right) t\right]+\left[\cos \left(\omega-\omega_{k}\right) t\right]\right) .
$$

The first term in relation (4) corresponds to elastic scattering of light without changing the frequency (Rayleigh scattering), the second - anti-Stokes scattering of light with frequency $\left[\left(\omega+\omega_{k}\right)\right]$, the third represents Stokes scattering of light with frequency $\left[\left(\omega-\omega_{k}\right)\right]$.

Within the framework of quantum-mechanical consideration of the phenomenon of Raman scattering, it is possible to obtain a relation for the ratio of the intensities of the Stokes and anti-Stokes light scattering, which satisfies the experimental data. In Placzek theory, for example, the following formula was obtained [6]

$$
\frac{I_{S}}{I_{a S}}=\frac{\left(\omega_{0}-\omega_{m n}\right)^{4}}{\left(\omega_{0}+\omega_{m n}\right)^{4}} \cdot \exp \left(\frac{\hbar \omega_{m n}}{k T}\right) .
$$

It is confirmed by experiments under conditions of thermal equilibrium of a thermodynamic system.

Disordered condensed media as randomly oriented systems, such as liquids, isotropic polymers and quasicrystalline systems are the most preferable for studying physical objects by the Raman scattering method. The classical explanation of the phenomenon of Raman scattering (RS) currently retains its fundamental importance [1-6].

\section{Influence of clustering processes on Raman scattering in liquids}

The dipole moment of a dimer in the cluster structure is determined by the number of particles in this cluster. For example, in [8, 9, 27], a formula was proposed according to which the dipole moment of a molecule in a water cluster is proportional to the square root of the number of particles in the cluster.

The induced dipole moment of the dimer in the cluster structure under the action of a harmonic electromagnetic wave is represented by the relation

$$
\mu_{\mathrm{dim}}=\bar{\alpha} E_{0} \sin \left(\omega_{0} t\right) .
$$

The average polarizability of a dimer formation $\bar{\alpha}$ depends on the number of particles in the middle cluster, which includes the dimer, and this dependence can be described by the formula [10] 


$$
\bar{\alpha}=\alpha_{0}\left[1+\frac{1}{3}\left(\frac{\bar{Z}}{\bar{Z}_{\max }}\right)\right]
$$

where $\bar{Z}$ is the average number of particles in a cluster, $\bar{Z}_{\max }$ is the maximum possible number of particles in a cluster for given parameters of the state of matter, $\alpha_{0}$ is the polarizability of a molecule in gases.

The average number of particles in a cluster in the liquid structure is determined by the state parameters, in particular, by the density of the liquid, and this dependence can be represented by the relation [11]

$$
\bar{Z}=\exp \left(\frac{\rho}{\rho_{C}}\right)
$$

where $\rho, \rho_{C}$ are the density and critical density of the liquid-vapor transition.

The maximum value of the average number of particles in the cluster is observed in the liquid at the maximum value of the ratio $\rho / \rho_{C}=3.06$ (for benzene), then $\bar{Z}_{\max }=21$.

Relation (7) was used to calculate the refractive index for simple and organic liquids on the liquid-vapor equilibrium line in a wide range of state parameters variation within the framework of the theory of effective polarizability of Frenkel L.I. and Gubanov A.I. [12]

$$
n^{2}=1+\frac{4 \pi}{v} \bar{\alpha}_{e f f}=1+\frac{4 \pi}{v} \alpha_{0}\left[1+\frac{1}{3}\left(\frac{\bar{Z}}{\bar{Z}_{\max }}\right)\right] .
$$

Calculations of the refractive index according to relation (9) and comparison of theoretical calculations with experimental data for liquid noble gases, cyclic hydrocarbons (arenes) and their halogen-substituted ones have shown that relation (7) is quite acceptable for assessing the average polarizability of a dimer formation in the middle cluster structure [10].

The value enclosed in a square bracket $\left[\frac{1}{3}\left(\frac{\bar{Z}}{\bar{Z}_{\max }}\right)\right]<<1$ is always less than one, therefore this square bracket in relations (7) and (9) can be considered as an expansion in a power series of some function, for example $\operatorname{tg}(x)$, and then we write

$$
\operatorname{tg}(x)=x+\frac{x^{3}}{3}+\frac{2 x^{5}}{15}+\ldots=\sum_{n=1}^{\infty} \frac{B_{2 n}(-4)^{n}\left(1-4^{n}\right)}{(2 n) !} \cdot x^{2 n-1}
$$

where $B_{2 n}$ are the Bernoulli numbers.

Considering only the first two terms in the expansion (10), we obtain

$$
\frac{\operatorname{tg}(x)}{x}=\left(1+\frac{1}{3} x^{2}\right)=\left[1+\frac{1}{3}\left(\frac{\bar{Z}}{\bar{Z}_{\max }}\right)\right] \text {, где } x=\sqrt{\left(\frac{\bar{Z}}{\bar{Z}_{\max }}\right)} .
$$

Having carried out obvious mathematical transformations, taking into account the fact that it is possible to take the approximation 


$$
\cos (x) \approx 1-\frac{x^{2}}{2}+\ldots \approx \frac{1}{1+x^{2} / 2}
$$

we get the ratio

$$
\frac{\operatorname{tg}(x)}{x}=\frac{\left(1+x^{2} / 2\right)}{x} \cdot \sin (x)=\frac{\bar{\alpha}}{\alpha_{0}} .
$$

Consequently, the average polarizability of a dimer in the middle cluster is determined by the formula

$$
\bar{\alpha}=\alpha_{0} \cdot \frac{\left(1+x^{2} / 2\right)}{x} \cdot \sin (x), \quad x=\frac{\omega_{k}}{\omega_{\max }}=\sqrt{\frac{\bar{Z}}{\bar{Z}_{\max }}} .
$$

The induced dipole moment according to the relations (11) and (14) is determined by the relation

$$
\mu_{\mathrm{dim}}=\alpha_{0} \frac{\left(1+x^{2} / 2\right)}{x} \cdot E_{0} \sin (x) \sin \left(\omega_{0} t\right)
$$

On the one hand the value of $x$ is determined by the average number of particles in a cluster; on the other hand, it is related to the frequencies of libration vibrations of dimer formations in the cluster structure, and the relation should be satisfied

$$
x=\omega_{k} t=\left(\omega_{\min } \sqrt{\bar{Z}}\right) t .
$$

Using the trigonometric identity ( $2 \sin a \cdot \sin b=[\cos (a-b)-\cos (b+a)])$, relation (15) can be written in the form

$$
\mu_{\operatorname{dim}}=\alpha_{0} \frac{\left(1+x^{2} / 2\right)}{2 x} \cdot E_{0}\left[\cos \left(\omega_{0}+\omega_{\min } \sqrt{\bar{Z}}\right) t-\cos \left(\omega_{0}-\omega_{\min } \sqrt{\bar{Z}}\right) t\right] .
$$

The obtained relation (17) coincides in its form with formula (4) of the classical theory of the Raman effect, but formula (17) has several important consequences. First, the integration of clustering processes in liquids leads to the dependence of the maxima of the spectral bands for the observed frequencies in the Raman spectra on the composition of cluster formations, and this dependence is determined by the formula

$$
\omega_{k}=\omega_{\min . k} \cdot \sqrt{\bar{Z}}_{k} .
$$

The minimum frequency of libration vibrations of a dimer formation in the cluster structure $\omega_{\min . k}$ is determined by the formation enthalpy $\Delta H_{\operatorname{dim} . k}$ and the inertia moment $J_{\text {dim.k }}$ of the dimer configuration [13]

$$
\omega_{\min . k}=\sqrt{\frac{2 \Delta H_{\operatorname{dim} . k}}{J_{\operatorname{dim} . k}}} .
$$


Polyatomic molecules of complex geometrical shape can form several possible configurations of dimers, differing in the inertia moment about the main axes and the energy of configuration formation. It has been established now that benzene molecules can form four types of dimeric configurations: Sandwich (S), T-shaped, parallel-displaced (PD), and Chain-configuration (CC) - configurations differing in the value of equilibrium distances between the centers of molecule mass, energy formation of the configuration (binding energy of the dimer) and the inertia moment about the axis passing through the center of configuration mass (Figure 1) [7, 14, 15].

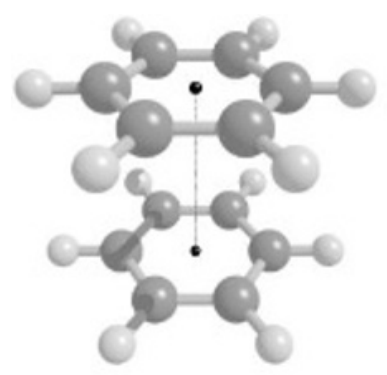

a)

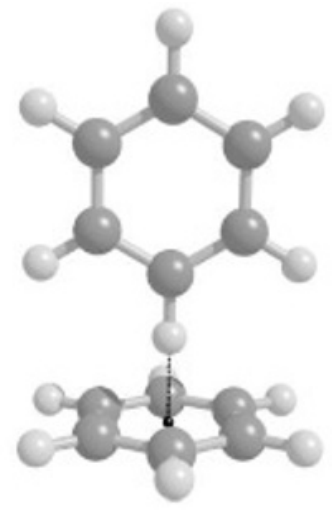

b)

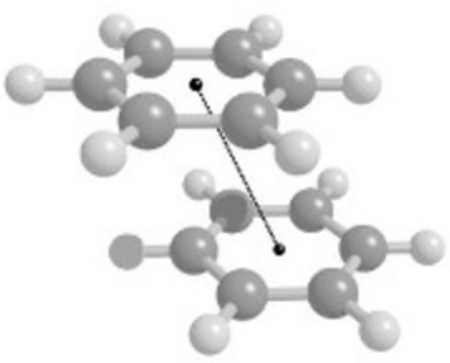

c)

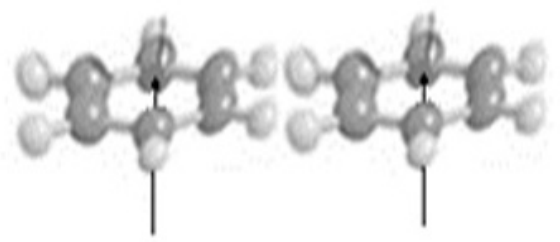

d)

Fig. 1. a) Sandwich (S), b) T-shape, c) Parallel displacement (PD), and d) Chain configuration (CC) of the benzene dimer configuration.

Thus, according to formula (19), for liquids with polyatomic molecules, there is a set of minimum frequencies, each of which forms a spectral series in the Raman spectrum of the liquid.

With a spontaneous breakup of a cluster, the number of particles in its composition changes and becomes equal $\bar{Z}_{j}$, and then the frequency of libration vibrations of the dimer in the newly formed cluster will be

$$
\omega_{j}=\sqrt{\frac{2 \Delta H_{\text {dim.k }}}{J_{\text {dim.k }}}} \cdot \sqrt{\bar{Z}_{j}} .
$$

The change in frequency is accompanied by the emission or absorption of a quantum of energy with a frequency

$$
\omega_{j k}=\sqrt{\frac{2 \Delta H_{\text {dim.k }}}{J_{\text {dim.k }}}} \cdot \sqrt{\bar{Z}_{i}}\left(1-\sqrt{\frac{\bar{Z}_{j}}{\bar{Z}_{k}}}\right) .
$$


To calculate the frequency of the emitted energy quantum during the spontaneous decay of the initial cluster, it is necessary to establish the decay law of cluster systems in a condensed medium, on the basis of which the correction root in the parenthesis of formula (21) can be calculated.

Assuming that the root in the parenthesis of this formula remains constant, we can put this value equal to the square root of the constant "golden ratio», i.e. put $\bar{Z}_{j} / \bar{Z}_{k}=\lim \left(F_{i-1} / F_{i}\right)=0.618 \ldots$, then formula (21) will be written in the form of the relation

$$
\omega_{j k}=0.2139 \sqrt{\frac{2 \Delta H_{\operatorname{dim} . k}}{J_{\operatorname{dim} . k}}} \cdot \sqrt{F_{j}},
$$

where $F_{i}=1,2,3,5,8,13,21,34,55, \ldots$ are the Fibonacci numbers.

Fibonacci clusters are most likely in the structure of disordered condensed media, and the number of particles in them coincides with the numbers of the successive Fibonacci series. Clusters with the number of particles from the Fibonacci series are the most stable in the structure of condensed media, as indicated by experimental data on mass spectroscopy of cluster fluxes of various substances [16]. The interaction of Fibonacci clusters of different composition leads to the formation of new cluster systems, which again represent Fibonacci clusters ultimately. Merging of two Fibonacci clusters can occur according to the scheme $[17,18]$

$$
F_{n+2}+F_{n-2}=F_{n}+F_{n}+F_{n}=3 F_{n} .
$$

As a result, a cluster is formed with the number of particles equal to three times the Fibonacci number, but such a cluster is unstable and splits into three identical Fibonacci clusters. For example, the most probable number of particles in an argon cluster near the melting point is $F_{n}=8$, therefore, according to formula (23), it is necessary to write $F_{n+2}=21 ; F_{n-2}=3 ; F_{n+2}+F_{n-2}=3 F_{n}=24$. A cluster with a number of particles equal to 24 is not among the most stable clusters according to the data of mass spectroscopic experiments [16] and splits into three identical Fibonacci clusters.

The merging of four or more Fibonacci clusters at the same time has a small probability; however, the scheme for implementing such a process can be easily obtained by generalizing formula (23) and presenting the decay process in the form

$$
F_{n-2}+F_{n}+F_{n+2}=4 F_{n} ; F_{n-2}+F_{n}+F_{n}+F_{n+2}=5 F_{n} .
$$

Each of the cluster formation schemes (24) leads to a constant «golden» section; therefore, they are derivatives of pair collisions and merging of clusters. If $F_{n}=8$, then $F_{n-2}+F_{n}+F_{n+2}=4 F_{n}=32$. A cluster with 32 particles, according to mass spectroscopy data, is a «magic» cluster [16], and it is relatively stable and may not decay.

Let us note one more scheme for the formation of cluster systems, when Fibonacci clusters are formed with the number of particles with consecutive Fibonacci numbers [17, 18]

$$
\sum_{i=1}^{n} F_{i}=F_{n+2}-1
$$

The proposed model of the formation of cluster systems makes it possible to explain the appearance of spectral bands in the Raman spectra of condensed media, which are caused 
by the processes of formation and decay of Fibonacci cluster systems in the structure of matter.

\section{Results of experimental verification of the cluster model using spectroscopic data}

To test the obtained relations, which take into account clustering processes in Raman scattering in liquids, we have chosen liquid benzene and toluene, which have great practical application; moreover, these liquids are model substances in theoretical studies and they are well studied.

Raman and IR spectra of liquid benzene in the frequency range $400-3300 \mathrm{~cm}^{-1}$ have been studied in detail; it suffices to mention the work of Bertie and Keefe in the frequency range 11.5-6200 $\mathrm{cm}^{-1}$ [19], Chelli at al. [20], Badoglu and Yurdakul [21]. In the crystalline state, Raman and IR spectra of benzene in the frequency range $55-3131 \mathrm{~cm}^{-1}$ were studied by Kearley, Jonson, Tomkinson [22]. Within the total errors, the spectral measurements of the authors of [19-22] are in good agreement with each other. Table 2 shows data on Raman spectra in the far spectral region for benzene, obtained by various authors.

The formation energy of various benzene dimeric configurations has been calculated by many authors using quantum chemistry methods using various interaction potentials. Some of the results of such calculations are shown in Table 1.

Having data on the formation energy of various dimeric configurations and their inertia moments with respect to the principal axes, one can calculate the minimum frequencies of libration vibrations of dimers in the cluster structure using the formulas

$$
\omega_{\min , i}^{\prime}=0.2139 \sqrt{\frac{2 \Delta H_{\mathrm{dim}, i}}{J_{\mathrm{dim}, i}}}, \quad \omega^{\prime \prime}{ }_{\min , i}=0.3820 \sqrt{\frac{2 \Delta H_{\mathrm{dim}, i}}{J_{\mathrm{dim}, i}}} .
$$

We have noted that the ratio of frequencies is $\kappa=\frac{\omega_{\min . j}^{\prime \prime}}{\omega_{\min . j}^{\prime}}=1.787 \ldots$, that is actually observed in the Raman spectra of the liquid.

Table 1. Binding energies and equilibrium distances of configurations of benzene dimers ( $E$ in $\mathrm{kcal} / \mathrm{mol}$ and $R$ in A) [23-26].

\begin{tabular}{|c|c|c|c|c|c|c|c|}
\hline \multirow{2}{*}{$\begin{array}{c}\text { Configurations of } \\
\text { the benzene dimer / } \\
\text { Basis [26] }\end{array}$} & \multicolumn{2}{|c|}{$\begin{array}{c}\text { Sandwich } \\
\boldsymbol{~}\end{array}$} & \multicolumn{2}{c|}{$\begin{array}{c}\text { T-shaped } \\
\boldsymbol{T}\end{array}$} & \multicolumn{3}{c|}{$\begin{array}{c}\text { Parallel-displaced } \\
\text { configurations } \\
\text { PD }\end{array}$} \\
\cline { 2 - 8 } & $\boldsymbol{R}$ & $\boldsymbol{E}$ & $\boldsymbol{R}$ & $\boldsymbol{E}$ & $\boldsymbol{R}_{\mathbf{1}}$ & $\boldsymbol{R}_{\mathbf{2}}$ \\
\hline 6-31+G*240 & -1.124 & 4.030 & -1.938 & 5.137 & -1.983 & 3.6 & 1.768 \\
\hline 6-31+G*-BF1f 262 & -1.996 & 3.870 & -2.809 & 4.958 & -3.017 & 3.4 & 1.705 \\
\hline aug-cc-pVDZ*c 336 & -2.830 & 3.750 & -2.998 & 4.958 & -4.123 & 3.4 & 1.582 \\
\hline $\begin{array}{c}\text { aug-cC-pVDZ-BF2 } \\
\text { 442 }\end{array}$ & -3.179 & 3.731 & -3.372 & 4.889 & -4.531 & 3.4 & 1.558 \\
\hline $\begin{array}{c}\text { EFP2 Monte } \\
\text { Carlo/simulated }\end{array}$ & -2.41 & 4.0 & -2.80 & 5.10 & - & - & - \\
\hline CCSD(T) & -1.76 & 3.90 & -2.73 & 5.0 & - & - & - \\
\hline The average & -2.81 & 3.767 & -3.122 & 4.929 & -4.081 & 3.418 & 1.596 \\
\hline
\end{tabular}


Table 2 shows the values of the minimum frequencies of libration vibrations of dimers in the structure of benzene clusters for various configurations.

Table 2. Minimum frequencies of libration vibrations of dimers of various configurations.

\begin{tabular}{|c|c|c|c|c|}
\hline Configuration & $\begin{array}{c}\Delta H_{\mathrm{dim} . i}, \\
\mathrm{kcal} / \mathrm{mol}\end{array}$ & $\begin{array}{c}J_{\mathrm{dim} . i} \cdot 10^{45} \\
\mathrm{~kg} / \mathrm{m}^{2}\end{array}$ & $\begin{array}{c}\omega_{\min }, \\
\mathrm{cm}^{-1},(19)\end{array}$ & $\begin{array}{c}\omega_{\text {Raman }}, \\
\mathrm{cm}^{-1},[19,20]\end{array}$ \\
\hline Chain-Config. (CC) & -3.50 & 11.72 & 14.5 & 11 \\
\hline Sandwich (S) & -2.02 & 5.86 & 15,6 & 15 \\
\hline Paral.-disp. (PD) & -2.44 & 7.33 & 15.3 & 15 \\
\hline T-shaped (T) & -2.82 & 4.40 & 21.2 & 22 \\
\hline Chain-Config. (CC) & -3.50 & 11.72 & 26.0 & 26 \\
\hline Sandwich (S) & -2.02 & 5.86 & 27.9 & 27 \\
\hline Paral.-disp. (PD) & -2.44 & 7.33 & 27.4 & 30 \\
\hline T-shaped (T) & -2.82 & 4.40 & 38.0 & 40 \\
\hline
\end{tabular}

Arranging the entire set of frequencies obtained by formulas (22) and (26) in ascending order, we obtain the Raman spectrum of benzene, recorded in experimental studies. Table 3 shows the results of theoretical calculations of the Raman spectrum frequencies according to the proposed method and comparison with the experimental data of various authors.

Table 3. Libration frequencies in the Raman spectrum of crystalline and liquid benzene.

\begin{tabular}{|c|c|c|c|c|c|c|c|}
\hline \multirow[b]{2}{*}{$F_{i}$} & \multicolumn{4}{|c|}{$\omega_{k}, \mathrm{~cm}^{-1},(22)$} & \multirow{2}{*}{$\begin{array}{c}\text { Calcu- } \\
\text { lations } \\
(27)\end{array}$} & \multicolumn{2}{|c|}{ Experiment } \\
\hline & $\begin{array}{c}\omega_{1 \mathrm{~min}}= \\
=11 \mathrm{~cm}^{-1}\end{array}$ & $\begin{aligned} & \omega_{2 \min }= \\
= & 21 \mathrm{~cm}^{-1}\end{aligned}$ & $\begin{aligned} & \omega_{3 \min }= \\
= & 28 \mathrm{~cm}^{-1}\end{aligned}$ & $\begin{aligned} & \omega_{4 \min }= \\
= & 38 \mathrm{~cm}^{-1}\end{aligned}$ & & {$[22]$} & [19] \\
\hline 2 & 15.5 & 29.7 & 39.6 & 53.7 & 31.1 & - & 31.4 \\
\hline 3 & 19.1 & 36.4 & 48.5 & 65.8 & 47.0 & - & 46 \\
\hline 5 & 24.6 & 47.0 & 62.6 & 85.0 & 53.7 & 55 & 53.5 \\
\hline 8 & 31.1 & 59.4 & 79.2 & 107.5 & 64.1 & 64 & - \\
\hline 13 & 39.7 & 75.7 & 101.0 & 137.0 & 85,0 & 85 & 86.6 \\
\hline 21 & 50.4 & 96.2 & 128.3 & 174.1 & 96.2 & 94 & - \\
\hline 34 & 64.1 & 122.4 & 163 & 222 & 101 & 102 & - \\
\hline 55 & 81.6 & 156 & 208 & 282 & 128.3 & 128 & - \\
\hline 89 & 104 & 198 & 264 & 358 & 168 & - & 168 \\
\hline 144 & 132 & 252 & 336 & 456 & 208 & - & 210 \\
\hline 233 & 168 & 321 & 427 & 580 & 264 & - & 264 \\
\hline 377 & 214 & 408 & 544 & 738 & 321 & - & 302 \\
\hline 610 & 272 & 519 & 692 & 939 & 408 & 405 & 403 \\
\hline 987 & 346 & 660 & 880 & 1194 & 456 & - & 447.6 \\
\hline 1597 & 440 & 840 & 1119 & 1510 & 692 & 690 & - \\
\hline
\end{tabular}


In the frequency range 30-350 $\mathrm{cm}^{-1}$, which corresponds to cluster formations with the number of particles from 2 to 55 in the structure of liquid benzene, there is a good agreement between the theoretical and experimental values of frequencies within the total comparison errors. In the frequency range 350-2000 $\mathrm{cm}^{-1}$, which corresponds to cluster formations of up to 1500 particles in their composition, the calculation error can reach $10 \%$, which can be explained by the fact that, firstly, vibration degrees of freedom of molecules can be excited at these frequencies, and secondly, the formation of clusters with more than 100 particles is unlikely in organic liquids, for such liquids the formation of small clusters is typical.

\section{Conclusion}

In the structure of organic liquids within the framework of the classical theory of Raman scattering the integration of clustering processes leads to the fact that the experimentally observed frequencies in the Raman spectrum are proportional to the square root of the number of particles in the most stable clusters. The appearance of spectral bands in Raman spectra (30-350 $\left.\mathrm{cm}^{-1}\right)$ can be explained by libration vibrations of dimer formations of various configurations in the cluster structure.

From a wide variety of clusters in the structure of liquids, a special class of clusters can be distinguished, called Fibonacci clusters, which differ in a number of features of their composition and internal structure. The sequential formation or collapse of a cluster with the number of particles from the Fibonacci series occurs according to the rule when the newly emerged cluster has the same number of particles from the series of Fibonacci numbers, and the ratio of the number of particles in two successively formed clusters, equal to the «golden» ratio $\Phi=1.618 \ldots$, is preserved.

\section{References}

[1] C.V. Raman, K.S. Krishnan, A new type of secondary radiation, Nature, v. 121, p. 501 (1928)

[2] C.V. Raman, A new type of secondary radiation, Nature, v. 121, p. 619 (1928)

[3] G. Landsberg, L. Mandelstam, Eine neue Erscheinung bei der Lichtzertreuung, Naturwissenschaften, v. B. 16, p. 557 (1928)

[4] V.S. Gorelik, Modern problems of Raman spectroscopy, Ed. M.M. Sushinsky (Nauka, Moscow, 1978)

[5] I.L. Fabelinsky, Prediction and discovery of the fine structure of the Rayleigh line, Uspekhi Fizicheskikh Nauk, v. 170, p. 93 (2000)

[6] G. Plachek, Rayleigh Scattering and Raman effect, Ed. prof. L. Rosenkevich (State scientific tech. publishing house of Ukraine, Kharkov, 1935)

[7] G.A. Melnikov, V.N. Verveyko, Yu.F. Melikhov, M.V. Verveyko, A.V. Polanski, Heat capacity and elastic characteristics of monoatomic and organic liquids with account for cluster formation, High Temperature, v. 50(2), p. 214, (2012)

[8] D.Yu. Dubov, A.A. Vostrikov, Dipole capture of a slow electron by a water cluster, JETP Letters, v. 86, pp. 454-457 (2007)

[9] A.A. Vostrikov, D.Yu. Dubov, S.V. Drozdov, Dipole moment of water clusters and the greenhouse effect, Letters in JETP, v. 34(5), pp. 87-94, (2008) 
[10] G.A. Melnikov, N.M. Ignatenko, A.V. Polyansky, The polarizability of molecules within the cluster model of matter, Bulletin of Kursk State University, no. 4(33), pp. 17-24 (2010)

[11] G.A. Melnikov, Heat of Melting of Small Clusters in the Model of the Potential with the Effective Well Depth, Physics of the Solid State, v. 60(5), pp. 1000-1004 (2018)

[12] Y.I. Frenkel, A.I. Gubanov, Current state of the theory of polarization of dielectrics, UFN, no. 24, pp. 68-121 (1940)

[13] G.A. Melnikov, N.M. Ignatenko, V.G. Melnikov, E.N. Cherkasov, O.A. Manzhos, The Structure of Small Cluster and IR Spectrum Condensed Matters, Journal of Nano- and Electronic Physics, v. 7(4), pp. 04087(3p.) (2015)

[14] Ding Y., at all, Journal of Computational Chemistry, v. 29(2), pp. 275 (2007)

[15] G.A. Melnikov, N.M. Ignatenko, V.M. Paukov, V.V. Suchilkin, Modeling of dimeric formations of benzene, Physico-mathematical modeling of systems. Materials of the XIX International seminar (VSTU, Voronezh, 2018)

[16] O. Echt, O. Kandler, T. Leisner, W. Miehle, E. Recknagel, Magic numbers in mass spectra of large van der Waals cluster, J. Chem. Soc. Faraday Trans., v. 86, pp. 2411 (1990)

[17] The Fibonacci Association, URL : http://www.mscs.dal.ca/Fibonacci/

[18] The Fibonacci Quarterly, URL : http://www.fq.math.ca/

[19] J.E. Bertie, C.D. Keefe, Infrared intensities of liquids XXIV: optical constants of liquid benzene-h6 at $25^{\circ} \mathrm{C}$ extended to $11.5 \mathrm{~cm}^{-1}$ and molar polarizabilities and integrated intensities of benzene-h6 between 6200 and $11.5 \mathrm{~cm}^{-1}$, Journal of Molecular Structure, v. 695-696, pp. 39-57, (2004)

[20] R. Chelli, G. Cardini, P. Procacci, at al., Simulated structure, dynamics, and vibrational spectra of liquid benzene, J. Chem. Phys., v. 113(16), pp. 6851 (2000)

[21] S. Badoglu, S. Yurdakul, FT-IR Spectroscopic and DFT Compu rational Study on Solvent Effects on S-Hydroxy-2-Quinolinecarboxylic Acid, Optics and spectroscopy, v. 118(3), pp. 385 (2015)

[22] G.J. Kearley, M.R. Jonson, J.Tomkinson, Intermolecular interactions in solid benzene, J. Chem. Phys., v. 124, pp. 044514 (2006)

[23] T. Smith, L.V. Slipchenko, M.S. Gordon, Modeling $\pi-\pi$ Interactions with the Effective Fragment Potential Method: The Benzene Dimer and Substituents, J. Phys. Chem. A., v. 112, pp. 5286-5294 (2008)

[24] A. Smith, M.S. Gordon, Benzene Pyridine Interactions Predicted by the Effective Fragment Potential Method, J. Phys. Chem. A, v. 115, pp. 4598-4609 (2011)

[25] D. Kim, Theoretical Study of the Formation of Benzene Excimer: Effects of Geometry Relaxation and Spin-state Dependence, Bull. Korean Chem. Soc., v. 35(9), 2014. http://dx.doi.org/10.5012/bkcs.2014.35.9.2738

[26] Y. Ding, Y. Mei, J.Z.H. Zhang, Fu-Ming Tao, Efficient bond function basis set for $\pi-\pi$ interaction energies, Journal of Computational Chemistry, v. 29(2), pp. 275279, (2007), DOI 10.1002/jcc.20788

[27] D.Yu. Dubov, A.A. Vostrikov, The absorption cross section of far infrared radiation by clustered water vapor, Letters in ZhTF, v. 36(4), pp. 54-60 (2010) 